\title{
Modeling framework of voltage-source converters based on equivalence with synchronous generator
}

\author{
Shulong TAN $^{1}$ (1) Hua GENG ${ }^{1}$, Geng YANG ${ }^{1}$, Huai WANG $^{2}$, \\ Frede BLAABJERG ${ }^{2}$
}

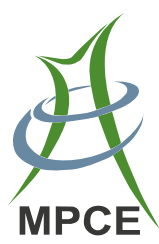

\begin{abstract}
Along with the increasing penetration of distributed generation with voltage-source converters (VSCs), there are extensive concerns over the potential virtual rotor angle stability, which is characterized by oscillations of power and frequency during the dynamic process of synchronization in the grid. Several control strategies have been developed for VSCs to emulate rotating inertia as well as damping of oscillations. This paper classifies these strategies and provides a small-signal modeling framework including all kinds of VSCs in different applications for virtual rotor angle stability. A unified perspective based on the famous Phillips-Heffron model is established for various VSCs. Thus, the concepts of equivalent inertia and the synchronizing and damping coefficients in different VSCs are highlighted, based on the similarities with the synchronous generator (SG) system in both physical
\end{abstract}

CrossCheck date: 17 May 2018

Received: 21 December 2017/Accepted: 17 May 2018/Published online: 23 July 2018

(C) The Author(s) 2018

$\triangle$ Shulong TAN

tansl_mail@foxmail.com

Hua GENG

genghua@tsinghua.edu.cn

Geng YANG

yanggeng@mail.tsinghua.edu.cn

Huai WANG

hwa@et.aau.dk

Frede BLAABJERG

fbl@et.aau.dk

1 Department of Automation, Tsinghua University, Beijing 100084, China

2 Department of Energy Technology, Aalborg University, Aalborg 9000, Denmark mechanisms and mathematical models. It revealed the potentiality of various VSCs to achieve equivalence with the SG. This study helps promote the unity of VSCs and traditional SGs in both theories and methods for analyzing the dynamic behavior and enhancing the stability. Finally, future research needs and new perspectives are addressed.

Keywords Voltage-source converter (VSC), Synchronous generator, Virtual rotor angle stability, Inertia,

Synchronizing, Damping

\section{Introduction}

Over the last decades, extensive use of grid-connected power electronic devices has brought changes into modern power systems. Various distributed generation (DG) units are connected to the alternate current (AC) grid via pulse width modulation (PWM) based voltage-source converters (VSCs) $[1,2]$. In addition, VSCs are also applied in gridconnected energy storage (ES) systems and are even used as commutation components in high-voltage DC (HVDC) transmission [3-5]. Consequently, VSCs are gaining a vital role in the modern power system.

From the perspective of the power system, VSC-based DG was originally expected to operate as a static synchronous generator (SSG) [6]. However, different from the traditional synchronous generator (SG), the VSC is regarded as a device of the flexible AC transmission system (FACTS), which is only responsible for exchanging controllable real and reactive power as an ancillary facility $[7,8]$ instead of bringing grid-forming capabilities into the power grid. Therefore, a considerable portion of VSCs are operated in current-source controlled (CC) mode, to keep the simplicity of the control strategy as well as an adequate 
utilization of renewable power [2]. From the perspective of emerging microgrid [9], control strategies are first proposed for parallel-connected VSC-based DGs in a standalone AC supply system [10], which is also motivated by the challenges in uninterruptible power supply (UPS) systems $[11,12]$. On this technical route, the VSC is regarded as an essential grid-forming component of the electrical grid, and it is operated in a voltage-source controlled (VC) mode. As an interface between DG systems and the grid, VSCs are endowed with similar responsibilities as the traditional SGs to deliver electric power into the AC grid. Nevertheless, VSCs lack rotating inertia and damping properties compared with the traditional SG. As the penetration level of VSC-based DG increases in modern power systems, traditional SGs, along with the inherent rotating inertia and damping properties to which they contribute, will be replaced on a large scale. The overall system inertia and damping are dramatically decreased, which can pose threats to grid dynamic performance and stability [13-15]. It is characterized by the low-frequency oscillations of power and frequency during various disturbances [14-17]. The oscillations may lead to over-current trip of the transmission line or even system disconnection [18]. Therefore, it is significant to investigate the stability of the modern power system with increasing VSCs.

Extensive research has focused on the stability analysis of the modern power system with VSCs, especially the small-signal stability analysis. Time-domain simulations are carried out to investigate the effects of the DGs, such as photovoltaic and wind turbine, on the dynamic behavior of the power system [19]. According to the time-domain simulation analysis, the intuitive dynamic response of a specific system can be presented and the optimal control design of a certain specific VSC can also be implemented, but the instability mechanism can hardly be explained, and the conclusion of generality is unavailable [20]. Eigenvalue analysis is another method to study the small-signal stability of the modern power system with VSCs [21-23]. For example, a detailed state space model is established for the entire system including droop-controlled VC-VSCs, network and loads in [22], and the eigenvalue analysis reveals that the droop coefficient will affect the low-frequency oscillation significantly. However, with the expansion of system scale, eigenvalue analysis based on detailed differential equations of the entire system has poor practicability in multi-VSC system because of the dramatically increased order of the differential equations [24]. The effect mechanism of a certain VSC parameter on the system oscillation is also not so clear [24]. Though there has been some preliminary theoretical research, a mature theoretical framework on the stability analysis of the modern power system with VSCs has not yet formed, compared with the traditional power system.
The oscillations of power and frequency come down to the rotor angle stability issue in the traditional power system $[25,26]$. Recently the concept of virtual rotor angle is proposed for VC-VSCs [25, 26]. Thus, it is expected to analyze the stability problem of the modern power system using the traditional method of SG-based power system, and then to establish the basis for methods of enhancing stability through VSC control. For instance, synchronization and stability mechanism of multi-VSC system is explored by utilizing a network of generalized Kuramoto oscillators, which is originally used to analyze the stability of traditional SG-based system [27]. This work is based on the classic Phillips-Heffron model [18, 28] of the droopcontrolled VC-VSC and is inspired by the equivalence between this VC-VSC and the traditional SG on the electromechanical time scale. It indicates that the well-developed theories as well as methodologies in traditional power system can be applied in the modern power system including SGs and increasing VSCs. The critical issue is that whether it is possible to establish the Phillips-Heffron model for various VSCs, since VSCs have diverse types because of the differences of the power interface on the AC side, the energy supply on the DC side and the specific control structure of the VSCs.

This paper provides a modeling framework for all kinds of VSCs for the small-signal stability analysis of the modern power system. Through exploring the equivalence between traditional SG and various VSCs on the electromechanical time scale, the Phillips-Heffron model is established for each kind of VSCs in this paper. Thus, the dynamics of VSCs are characterized the by using the inertia, synchronizing and damping coefficients (namely $K_{J}, K_{S}$ and $K_{D}$ ), the same as traditional SGs. It is beneficial for a clear understanding of various VSCs from the perspective of traditional SGs and power system. It is also expected to promote the application of the well-developed theories and methods in traditional power system to investigate the dynamic behaviors and stability issues of the modern power system with VSCs.

\section{Classic Phillips-Heffron model}

Power system stability is a complex subject, and classification is an effective method to deal with the complexities. Thus, there are rotor angle stability, namely the synchronization stability, frequency stability and voltage stability [29]. The rotor angle stability refers to the ability of interconnected power generation units to remain in synchronism. It is categorized as short-term phenomena that involves the electromechanical oscillations in power systems. Different from the frequency stability, which depends on the balance between generation and load in the 
mid or long term, the rotor angle stability is mainly associated with the inertia and damping, which are the concepts of the dynamic in the short term. Though the small-signal stability analysis is based on the linearization method, it is also validated to be effective for the nonlinear dynamic respond in most practical cases [18]. Furthermore, it is suitable for the modern power system with various small disturbances since its analysis result only depends on the initial state of the system.

To investigate the small-signal rotor angle stability, an appropriate model needs to be established. On the one hand, an accurate model to depict complete dynamics of a real SG is complicated. It is a seventh-order mathematical model comprising two parts: a second order mechanical model and a fifth-order electrical model [18]. Though the accurate model possesses a certain merit of theoretical research, the high-order mathematical model is difficult to implement and its practicability is limited. Therefore, simplified reduced-order models of SG are widely used in practical application according to the dynamics of concern and different accuracy requirements. In terms of rotor angle stability, the transient and sub-transient of electromagnetic dynamics in a real SG can be ignored. On the other hand, some ancillary components in an SG system can also be neglected in the rotor angle stability analysis.

For an SG-based power system, the inertia property, the synchronizing ability and the damping of electromechanical oscillations are critical features affecting the rotor angle stability. These aspects can be characterized by the classical Phillips-Heffron model. It reflects the small-signal dynamic relationship of the rotor angle and the angular frequency clearly and intuitively, which is applied as an effective tool for stability analysis and control. As shown in (1), the Phillips-Heffron model is given according to the block diagram in Fig. 1.

$\left\{\begin{array}{l}K_{J} \frac{\mathrm{d} \Delta \omega}{\mathrm{d} t}=-K_{S} \Delta \delta-K_{D} \Delta \omega \\ \frac{\mathrm{d} \Delta \delta}{\mathrm{d} t}=\omega_{0} \Delta \omega\end{array}\right.$

where $\omega$ is the angular velocity of the SG rotor; $\omega_{0}$ is the rated angular velocity; $\delta$ is the rotor angle. It is worth mentioning that the torques $T_{m}$ and $T_{e}$ in Fig. 1 can also be replaced by the corresponding power $P_{m}$ and $P_{e}$ in the per-

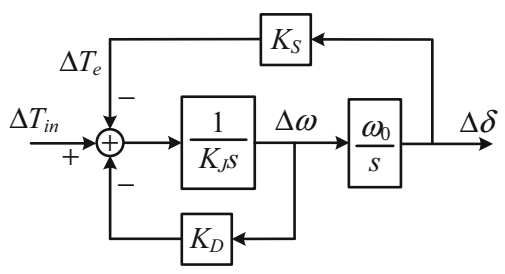

Fig. 1 Block diagram of Phillips-Heffron model unit system. The corresponding model parameters are listed as:

$\left\{\begin{array}{l}K_{J}=2 H \\ K_{S}=\frac{3}{2} \frac{U_{S G} U_{g}}{X_{L}} \cos \delta_{0} \\ K_{D}=D\end{array}\right.$

where $H$ is the inertia constant and $D$ is the damping torque coefficient, as described in [18]; $U_{S G}$ is the terminal voltage of the SG; $\delta_{0}$ is the steady-state rotor angle. As for the traditional SG, a single machine infinite bus system is usually regarded as the research paradigm [18], with a transmission line reactance $X_{L}$ and a bus voltage $U_{g}$.

\section{Classification of VSCs}

In order to enhance the frequency support and oscillation damping of the modern power system with increasing VSCs, some SG-imitation control strategies are proposed gradually for some certain VSCs to emulate the rotating inertia and damping of oscillations. For example, the VSYNC project [30] and the virtual synchronous generator (VSG) control [31]. Nevertheless, there are many types of VSCs according to different applications and control strategies. It is necessary to establish the Phillips-Heffron model for each type. Before presenting the modeling framework for all kinds of VSCs, the various VSCs are classified according to their low-frequency dynamics, which are associated with the oscillations of power and frequency.

As a power interface, VSC determines the source property of the DG from the perspective of the grid. Its interface control mode on the AC side will directly influence the interaction of the VSC and the grid. Thus, the control mode on the AC side of the VSC should be considered when modeling for stability analysis on the electromechanical time scale. Besides that, the character of the power supply on the DC side of the VSC will also impact the power dynamic of the VSC and then impact the design of the control loops. So, it is necessary to take the energy supply on the DC side into account when modeling for the stability analysis.

The AC interface control can be flexibly designed, such as the PQ control, droop control, VSG control and so on. According to the features of electric source, VSCs with these different control methods can be categorized as VCVSCs and CC-VSCs [32], as shown in Fig. 2. For the VCVSCs, the terminal voltage of the AC interface is regarded as the control target, and they regulate the frequency and amplitude of the terminal voltage to exchange the specified active power and reactive power with the grid. For the CCVSCs, the output current of the AC interface is taken as the 


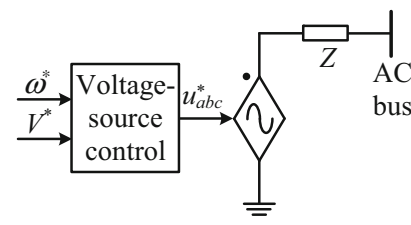

(a) VC-VSC

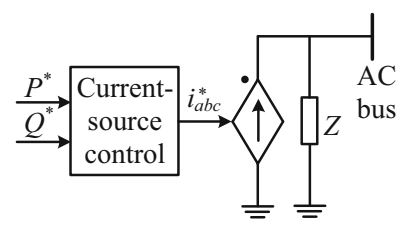

(b) CC-VSC
Fig. 2 VC-VSC and CC-VSC

control target, and they adjust the active component and reactive component of the output current for purpose of exchanging the corresponding power with the grid.

The energy supplies on the DC side of VSCs include photovoltaic panels, fuel cells, ESs, gas turbines, wind turbines, among others $[2,7]$. The VSCs with fuel cells or the ESs on the DC side are regarded as voltage-source supplied (VS) VSCs in this investigation. On the one hand, a DC/DC converter is always equipped with these energy supplies to stabilize the voltage of the DC capacitor. On the other hand, these energy supplies can provide sufficient energy for the VSC within a certain period of time. Those VSCs with photovoltaic panels, wind turbines, or gas turbines on the DC side are called power-source supplied (PS) VSCs in this paper. The wind turbine or gas turbine is connected to the VSC through a rectifier generally, and the stabilization of the DC capacitor voltage is in the charge of VSC itself. Though the photovoltaic panel is also connected to the VSC by a DC/DC converter, it cannot ensure adequate energy supply for the VSC under variable loads owing to the random energy supply of the photovoltaic panel. Therefore, VSCs with these energy supplies are called PS-VSC in terms of the energy supply on the DC side.

Taking both the control mode of the grid interface on the AC side and the energy supply on the DC side into account, various VSCs are classified into 4 categories. Each of them has some engineering application cases.

There are some VSCs used as the master in the microgrid with the master-slave control structure [9], and some VSCs used as the grid interface in the UPS system [33, 34]. Usually, these VSCs are equipped with ESs or fuel cells on the DC side, and their grid interface is VC mode. Thus, this type of VSCs is called voltage-source supplied and voltage-source controlled VSC (VS/VC-VSC).

For the VSCs with photovoltaic panels or wind turbines on the DC side, their grid interface is CC mode in most instances, such as the ordinary photovoltaic applications and wind power applications $[8,13,14]$. This kind of VSCs is named as power-source supplied and current-source controlled VSC (PS/CC-VSC).

For the CC-VSCs with photovoltaic panels or wind turbines, ES can also be equipped on the DC side to implement the virtual inertia for frequency supporting function of the CC-VSCs [30, 35]. These VSCs are called voltage-source supplied and current-source controlled VSC (VS/CC-VSC).

When the gas turbine or HVDC bus that is regarded as the power-source instead of voltage-source is configured on the DC side of the VSC, the grid interface on the AC side is controlled in the $\mathrm{CC}$ mode generally. However, in some engineering cases the grid interface is also operated in the VC mode [36]. These VSCs is named as power-source supplied and voltage-source controlled VSC (PS/VCVSC).

\section{Modeling framework of VSCs}

As mentioned above, an appropriate model for various VSCs is necessary to analyze the small-signal stability. Though there are several SG-imitation control strategies for some certain VSCs, a modeling framework for all VSCs is still not formed. From the perspective of the equivalence between the VSCs and the traditional SG, a modeling framework based on the classic Philips-Heffron model is established, as shown in Fig. 3.

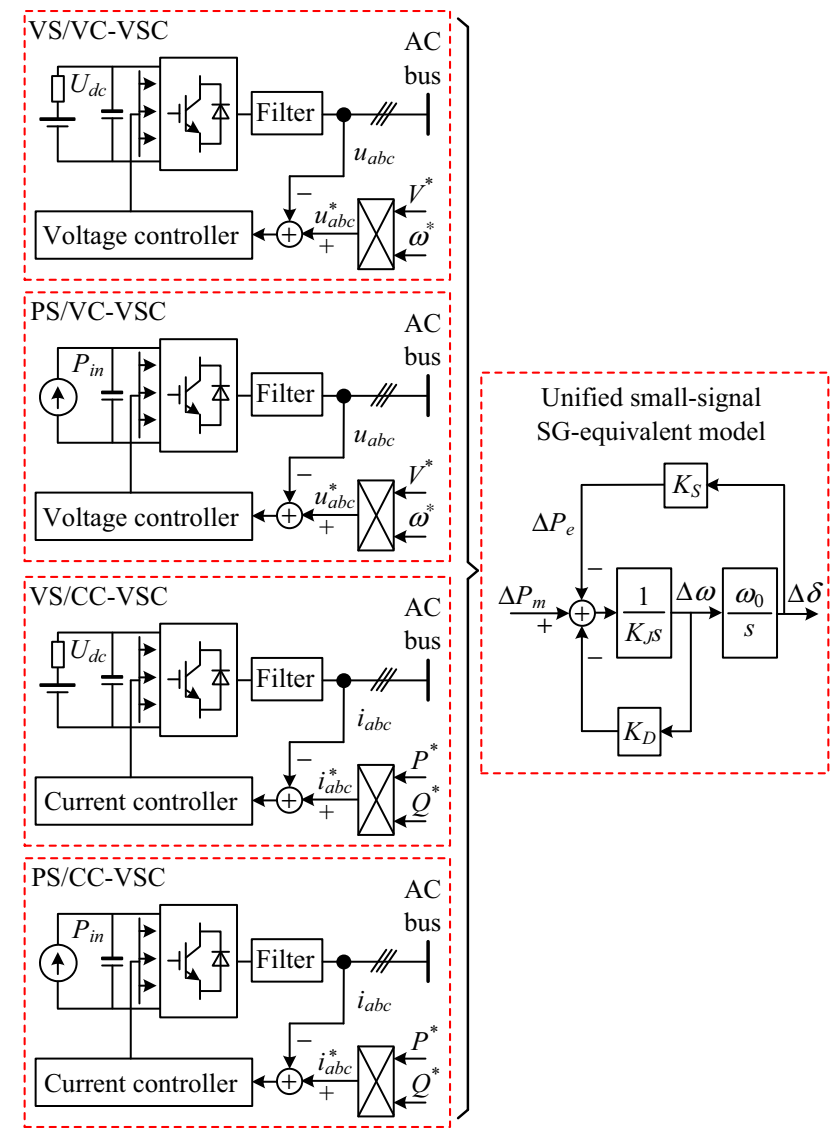

Fig. 3 SG-equivalent modeling framework of VSCs 
The necessity of this framework has several aspects. First, there are considerable challenges in the modeling and analysis of the modern power system with various VSCs as they have different structures and control modes. It is necessary to convert various VSCs into a unitary PhilipsHeffron model, which is beneficial to the simplification of the modeling and analysis. Second, there is no well-developed method for stability analysis of the modern power system with VSCs, it is necessary to apply the developed theory of the traditional power system on the modern power system with VSCs based on the equivalence between VSCs and the SG. Third, the integration and interaction of VSCs and SGs in the modern power system also put forward urgent requirements of the equivalence research between VSCs and the SG.

In addition, the SG-equivalent modeling of VSCs is feasible. From the perspective of the function, VSCs act as the grid interface of power generation, which is the same as the SG. From the perspective of the time scale, some important low-frequency dynamics of VSCs are on the same timescale as the electromechanical dynamics of the SG. It is possible to establish SG-equivalent model for VSCs using these low-frequency dynamics.

\subsection{SG-equivalent model of VS/VC-VSC}

As shown in Fig. 4, the DC capacitor voltage of the VS/ VC-VSC is controlled constant, and the output voltage on the AC side is taken as the control target. The control structure mainly includes a power-control outer loop and a voltage-control inner loop. Generally, the time scale of the power-control dynamic is about $3-10 \mathrm{~Hz}$, and the time scale of the voltage-control dynamic is about $200-400 \mathrm{~Hz}$ [22]. According to the perturbation theory [18], the powercontrol dynamic that is closer to the electromechanical time scale is the main consideration when implementing SG-equivalent modeling for the VS/VC-VSC.

There is a traditional power-control method for the VS/ VC-VSC, which is called droop control [10], as shown in

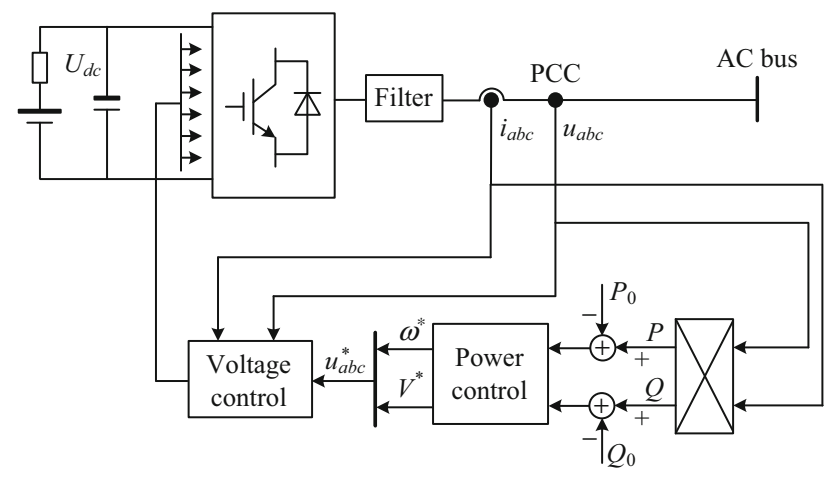

Fig. 4 Block diagram of VS/VC-VSC

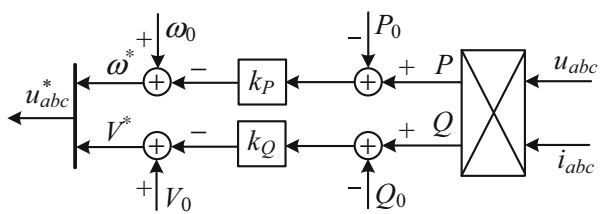

Fig. 5 Block diagram of traditional droop control

Fig. 5. It has been widely used since 1993, and has become a classical method for VS/VC-VSCs. For the common inductive network, droop-controlled VSCs take the responsibility of power sharing and balancing in electrical grids based on the frequency- and voltage-drooping mechanisms according to (3) [10]:

$\left\{\begin{array}{l}\omega^{*}=\omega_{0}+k_{P}\left(P-P_{0}\right) \\ V^{*}=V_{0}+k_{Q}\left(Q-Q_{0}\right)\end{array}\right.$

where $\omega^{*}$ is the reference value for angular frequency; $V^{*}$ is the reference value for voltage; $k_{P}$ and $k_{Q}$ are the droop gains for the active and reactive power control, respectively. In this paper, only the active power control is studied, since the reactive power control is almost identical.

In order to improve the virtual rotor angle stability of the microgrid under disturbances, a modified droop control for VSCs is proposed in [37]. As shown in Fig. 6, the droop gain is modified as a function of $\mathrm{d} \omega / \mathrm{d} t$. Thus, the equivalent inertia will be added virtually to the VSC by modifying the droop gain. Moreover, the VSC will supply higher power when the frequency deviation is large, which is beneficial to maintain the virtual rotor angle stability.

This SG-imitation strategy can be incorporated into the classical Phillips-Heffron model. The corresponding inertia coefficient, synchronizing coefficient, and damping coefficient can be derived as the following:

$\left\{\begin{array}{l}K_{J}=\frac{k_{1} \Delta P}{k_{P}} \\ K_{S}=\frac{3}{2} \frac{U_{1} U_{g}}{X_{L}} \cos \theta_{0} \\ K_{D}=\frac{1}{k_{P}}\end{array}\right.$

where $U_{1}$ is the terminal voltage; $\theta_{0}$ is the steady-state power angle; $\Delta P$ is the active power deviation; $k_{1}$ is the differential gain coefficient in Fig. 6. As shown in (4), the

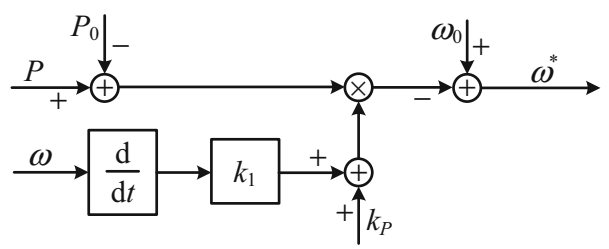

Fig. 6 Block diagram of modified $P-\omega$ droop control 
inertia or damping coefficient can be flexibly designed for VSCs without the physical constraint of a real SG. For example, the equivalent inertia in (4) is a variable instead of a constant, and is proportional to the active power deviation.

To further simulate the inertia and damping of the SG, a virtual inertia frequency control strategy is proposed in [38]. As shown in Fig. 7, a control block diagram is designed imitating the second order dynamic characteristics of the SG.

Under the guidance of the small-signal SG-equivalent modeling framework, classical Phillips-Heffron model can also be established for the VSC with virtual inertia frequency control strategy. The corresponding characteristic coefficients can be derived as the following:

$$
\left\{\begin{array}{l}
K_{J}=J_{1} \\
K_{S}=\frac{3}{2} \frac{U_{1} U_{g}}{X_{L}} \cos \theta_{0} \\
K_{D}=D_{p 1}+\frac{1}{k_{P}}
\end{array}\right.
$$

where $J_{1}$ is the inertia coefficient and $D_{p 1}$ is the original damping coefficient in [38].

Improved droop control strategies have achieved good SG-equivalent properties. However, it is not enough to simulate all the operation characteristics of SGs. If a VSCbased DG is operated as an SG completely, no difference between the VSC and SG can be detected from the grid side. Thus, some literature $[31,39,40]$ prepares to mimic the mechanical equations and the electromagnetic equations of a conventional SG in detail, and to construct the control model for the DG inverter. This makes the DG inverter analogous to the SG not only in external characteristics but also in the internal mechanism. These control strategies are called VSGs or synchronverters. Different from the previous concept of VSG for CC-VSCs, they are real voltage sources, and they achieve the unification of VSC-based DGs and SGs from the perspective of power system.

As shown in Fig. 8, the popular VSG scheme is proposed in [31]. $J_{2}$ is the moment of inertia of all the parts rotating with the rotor, $D_{p 2}$ is a damping factor, and $e$ is the back EMF due to the rotor movement. $T_{e}$ is the electromagnetic torque that is found from the energy stored in the machine magnetic field and the description of other

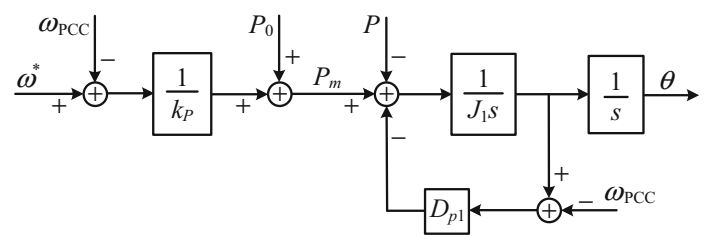

Fig. 7 Block diagram of virtual inertia frequency control

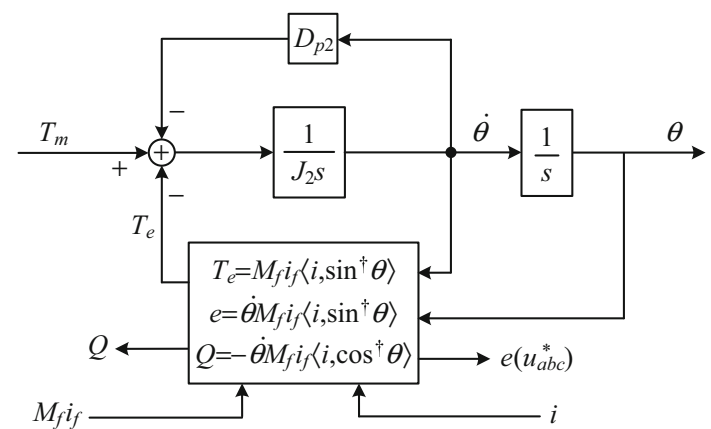

Fig. 8 Electronic part of a synchronverter

variables can also be found in [31]. When the VSG scheme is incorporated into the classical Phillips-Heffron model, the corresponding characteristic coefficients can be derived as the following:

$$
\left\{\begin{array}{l}
K_{J}=J_{2} \\
K_{S}=\frac{3}{2} \frac{U_{1} U_{g}}{X_{L}} \cos \theta_{0} \\
K_{D}=D_{p 2}
\end{array}\right.
$$

Besides the SG-imitation by deliberately constructing supplementary control loop, the inherent feature of droop control can also be utilized for SG-imitation. A block diagram illustrating the implementation of the frequencydroop control is shown in Fig. 9. In general, the active power measured at the grid interface of the power electronic converter is low-pass filtered. Based on the droop control, some literature explored its dynamic property $[21,22]$. The stability and the dynamics of the parallel-connected VC-VSC system are revealed to be strongly influenced by the value of the droop coefficients and by the cut-off frequency of the low-pass filter (LPF), which makes the bandwidth of the power flux controller much smaller than that of the voltage-control loop in the inverter. The filtering time constant affects the frequency response of the VSC remarkably $[21,22]$ and it is similar to the inertial time constant of a traditional SG [41, 42].

In recent years, the equivalence between the droopcontrolled VSCs and traditional SG is revealed on the electromechanical time scale [41, 42]. Assuming a constant set-point $\omega_{0}$ for the grid angular frequency and a constant reference $P_{0}$ for the active power, it is revealed that the droop gain $1 / k_{P}$ for the VSC is equivalent to the damping coefficient in SG system, and the filter time constant is

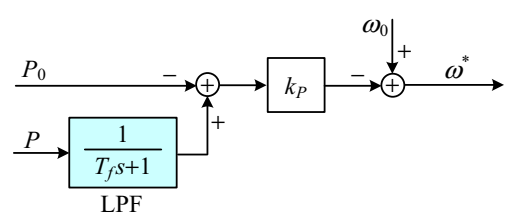

Fig. 9 Improved frequency-droop control using an LPF 
related to the rotor inertia in SG system. This SG-imitation can be categorized into the proposed modeling framework, and it can also be expressed by the block diagram as shown in Fig. 1. The corresponding characteristic coefficients can then explicitly be expressed by (7) and $T_{f}$ is the filtering time constant in Fig. 9.

$$
\left\{\begin{array}{l}
K_{J}=\frac{T_{f}}{k_{P}} \\
K_{S}=\frac{3}{2} \frac{U_{1} U_{g}}{X_{L}} \cos \theta_{0} \\
K_{D}=\frac{1}{k_{P}}
\end{array}\right.
$$

Strictly speaking, the grid angular frequency $\omega_{g}$ should be a measured parameter to provide the synchronous frequency, instead of being regarded as a constant value. Therefore, an improved method is implemented in [43], and the control diagram is shown in Fig. 10. Considering the variation of grid angular frequency $\omega_{g}$, a well-designed first-order lead-lag-unit is inserted to the droop control in order to generate the equivalent inertia and damping as SG. The VSC with the improved method in [43] can also be incorporated into the classical Phillips-Heffron model, the characteristic coefficients are given below, where $T_{a}$ is the lead time constant and $T_{b}$ is the lag time constant as shown in Fig. 10.

$$
\left\{\begin{array}{l}
K_{J}=\frac{T_{b}}{k_{P}} \\
K_{S}=\frac{3}{2} \frac{U_{1} U_{g}}{X_{L}} \cos \theta_{0} \\
K_{D}=K_{S} T_{a}
\end{array}\right.
$$

The equivalent SG model of droop-controlled VC-VSC has been successfully applied to synchronization and stability analysis for microgrids with multiple VSCs [44], using the classic Kuramoto oscillator theory, which is originally applied to analyze the stability of multi-SG systems [27].

\subsection{SG-equivalent model of the PS/VC-VSC}

The aforementioned equivalence between the droopcontrolled VSC and SG has been demonstrated, considering the DC-link as an ideal battery with infinite energy. Actually, the DC-link voltage may not maintain a constant in many actual circumstances without infinite energy on the DC side. Therefore, these VSCs should be regarded as PS/

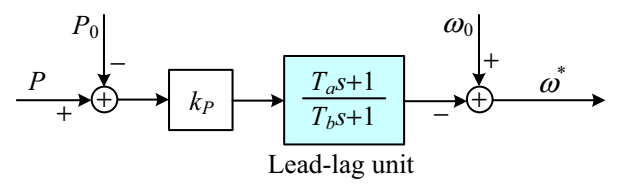

Fig. 10 Droop control with a first-order lead-lag unit
VC-VSCs and the DC-link dynamics should be considered. As shown in Fig. 11, the AC voltage reference of the inner control loop depends on the output of the power-control loop, which is responsible for maintaining the stable DC voltage.

Taking the DC-link dynamics of the PS/VC-VSC into account, emulating the behavior of traditional SG is also achieved, because the DC-link can be regarded as a virtual rotor of the SG. A new control strategy for PS/VC-VSCs is presented, which enables DC-link voltage regulation by frequency and power angle adjustment [36, 45-47]. As shown in Fig. 12, the cascaded frequency, angle, and DClink voltage loops are designed. Then additional damping and synchronizing properties are emulated in the VSC as in traditional SGs, and the DC-link dynamic is also considered. The electromechanical dynamics can be obtained in the following:

$J_{3} \frac{\mathrm{d} \omega}{\mathrm{d} t}=-K_{u 1} K_{u 2}\left(\omega-\omega_{0}\right)-K_{u 2} \theta+U_{D C}^{2}-U_{D C, \text { ref }}^{2}$

where $J_{3}$ is the virtual inertia associated with the DC capacitor; $U_{D C}$ and $U_{D C \text {,ref }}$ are the filtered value of the DClink voltage $u_{d c}$ and its reference value; $K_{u 1}$ and $K_{u 2}$ are the damping coefficients, $K_{u 2}$ can be interpreted as the synchronizing coefficient.

This SG-imitation can also be integrated into the proposed modeling framework, and the corresponding characteristic coefficients are expressed by (10).

$\left\{\begin{array}{l}K_{J}=J_{3} \\ K_{S}=\frac{3}{2} \frac{U_{1} U_{g}}{X_{L}} \cos \theta_{0} \\ K_{D}=K_{u 1} K_{u 2}\end{array}\right.$

In addition to the research that artificially constructed the virtual electromechanical swing dynamic of a traditional SG, some researches make use of the physical similarity between the DC-link capacitor and the SG rotor. The intrinsic electromechanical swing dynamic of DC-link capacitor is exploited to implement SG-imitation of VSCs. A steady-state linear relationship between the DC-link

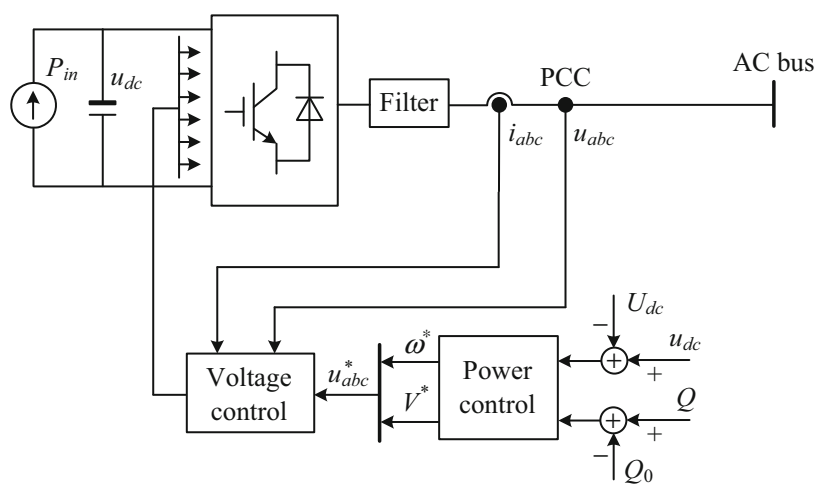

Fig. 11 Block diagram of PS/VC-VSC 


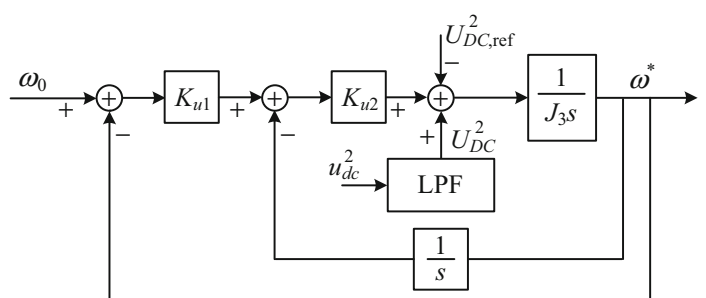

Fig. 12 Frequency-control scheme considering DC-link dynamics

voltage $u_{d c}$ and the angular frequency $\omega$ is also designed, which can be regarded as a generalized droop control between $u_{d c}$ and $\omega$ [48]. It implements the SG-imitation for PS/VC-VSCs by using the DC-link dynamics. Then a differentiator can be inserted to achieve the damping property in a dynamic, as shown in Fig. 13. The dynamic behaviors of the DC-link capacitor and the SG rotor are similar pattern as stated in [24]. The angular velocity of the SG rotor $\omega$ and the voltage of the DC capacitor $u_{d c}$ are corresponding variables. Thus, the corresponding dynamics can be obtained, as shown in (11).

$\omega^{*}=\omega_{0}+\left(1+s K_{d 1}\right)\left(u_{d c}-U_{d c}\right)$

The DC-link capacitor is similar to the SG rotor, and both of them are responsible for storing and transmitting transient energy. The DC-link voltage dynamic is expressed in (12).

$K_{J} \frac{\mathrm{d} u_{d c}}{\mathrm{~d} t}=P_{d c}-P_{s}$

where $P_{d c}$ is the active power that is injected into the DClink capacitor; $P_{s}$ is the active power that is delivered to the AC grid. Assuming that $P_{d c}$ keeps constant during a short time, the classical Phillips-Heffron model can also be derived according to the PS/VC-VSC in Fig. 13, and the corresponding characteristic coefficients can be listed in (13). As shown in (13), the equivalent inertia coefficient $K_{J}$ is related to the DC capacitance $C$, the rated DC voltage $U_{d c}$ and the rated active power $P_{\text {base }}$.

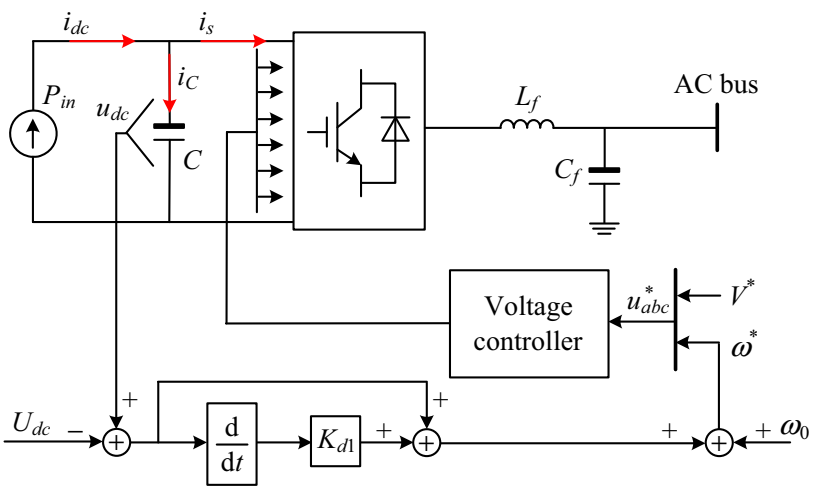

Fig. 13 Control scheme of frequency and DC-link voltage regulation
$\left\{\begin{array}{l}K_{J}=\frac{C U_{\mathrm{dc}}^{2}}{P_{\text {base }}} \\ K_{S}=\frac{3}{2} \frac{U_{1} U_{g}}{X_{L}} \cos \theta_{0} \\ K_{D}=K_{S} K_{d 1}\end{array}\right.$

\subsection{SG-equivalent model of VS/CC-VSC}

For the VS/CC-VSC, a DC/DC converter is often equipped to stabilize the voltage of the DC-link, and the energy supply on the DC side is able to provide sufficient energy for the VSC within a certain period of time. Since the voltage on the DC side is stable, the VSC control is focused on the output AC current control, as shown in Fig. 14. The VS/CC-VSC adjusts the active component and reactive component of the output $\mathrm{AC}$ current in order to exchange the corresponding power with the grid, with the help of the phase-locked loop (PLL).

By revealing the inherent PLL feature of the traditional SG, as shown in Fig. 15, a special PLL imitating the inherent phase-locking dynamic of SG is applied, instead of the conventional PLL with proportional-integral (PI) controller [49].

Based on the research achievement in [49], the VS/CCVSC with the special PLL can be integrated into the proposed modeling framework, and the corresponding characteristic coefficients are given in (14), where $J_{4}$ is the inertia coefficient and $K_{F}$ is the feedback gain as shown in Fig. 15.

$\left\{\begin{array}{l}K_{J}=J_{4} \\ K_{S}=U_{g} \cos \theta_{0} \\ K_{D}=k_{F}\end{array}\right.$

With a certain amount of ES connected to the DC-link, grid coupled VSCs are able to achieve virtual inertia emulation and also primary frequency control, thus the VSYNC project is put forward [30]. For the VS/CC-VSC,

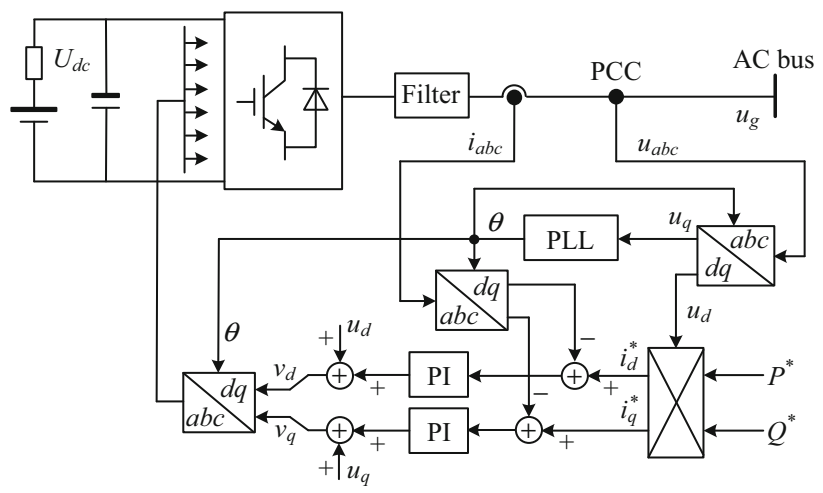

Fig. 14 Block diagram of VS/CC-VSC 


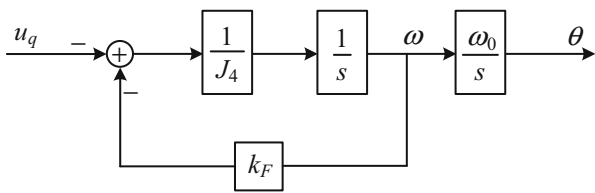

Fig. 15 Inherent PLL feature of traditional SG

some supplementary power-control loops are constructed to implement the virtual inertia and frequency response, due to the inherent output power margin on the DC side $[30,35]$. As shown in Fig. 16, $S_{\text {SOC }}$ is the state of charge (SOC) of the ES cell. The original power reference is $P_{\text {SOC,ref, }}$, which is generated merely according to the SOC. The inertia-imitating power reference $P_{i n \text {,ref }}$ and the frequency-adjusting power reference $P_{f \text {,ref }}$ is generated to implement the dynamics of traditional SG.

Under the guidance of the small-signal SG-equivalent modeling framework, classical Phillips-Heffron model can also be established for the VS/CC-VSC with supplementary power-control loops. The corresponding characteristic coefficients can be derived as the following:

$$
\left\{\begin{array}{l}
K_{J}=K_{\text {inertial }} \\
K_{S}=\frac{3}{2} \frac{U_{1} U_{g}}{X_{L}} \cos \theta_{0} \\
K_{D}=K_{f 1}
\end{array}\right.
$$

where $K_{f 1}$ and $K_{\text {inertial }}$ are the proportional gain coefficient and the differential gain coefficient, respectively.

Some researches $[50,51]$ further put forward that it is necessary for the VS/CC-VSC to completely mimic the static and dynamic properties of synchronous machine by making full use of its own dynamic advantage. Then the VSC can be regarded as conventional power stations from a grid point of view. The virtual synchronous machine (VISMA) model is first presented in [51], which is based on the complete two-shaft model of an electrically excited synchronous machine. As shown in Fig. 17, the voltages at the point of common coupling of the grid are measured to calculate the phase currents in strict accordance with the mathematical model of SG. Then these obtained phase currents are regarded as the reference for the VSC. Hence, seen from the grid side, the VSC can behave like a real SG

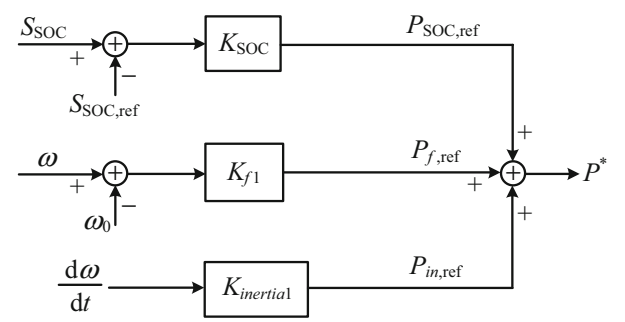

Fig. 16 Power-control loops are constructed to imitate the SG

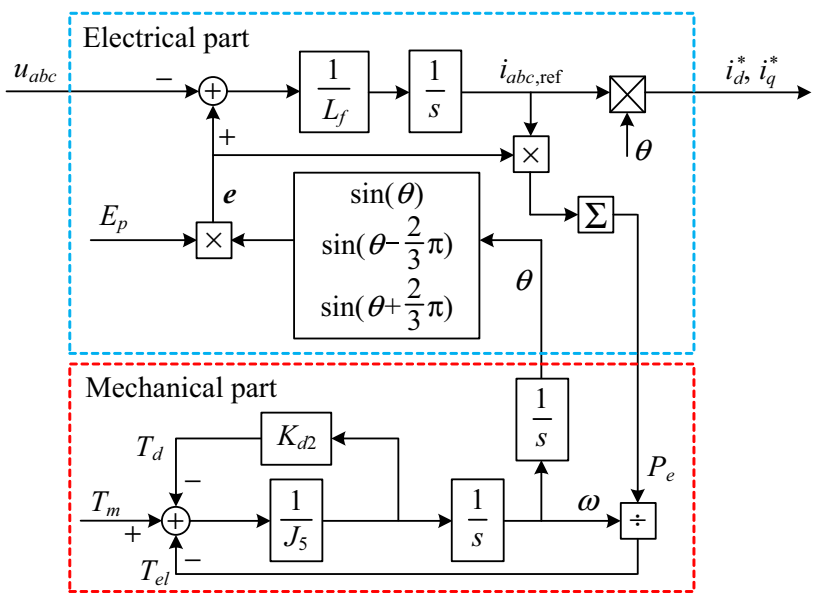

Fig. 17 VISMA control method

if it is always capable of feeding the current value calculated by the VISMA model.

The control strategy shown in Fig. 17 includes the electrical part and the mechanical part. The mechanical part is the main consideration when implementing SGequivalent modeling since its time scale is closer to the electromechanical time scale. Thus, the VS/CC-VSC with the VISMA control can also be integrated into the proposed modeling framework, and the corresponding characteristic coefficients are given in (16), where $J_{5}$ is the inertia coefficient and $K_{d 2}$ is the damping coefficient.

$\left\{\begin{array}{l}K_{J}=J_{5} \\ K_{S}=\frac{3}{2} \frac{U_{1} U_{g}}{X_{L}} \cos \theta_{0} \\ K_{D}=K_{d 2}\end{array}\right.$

For the VS/CC-VSC with short-term ES, the DC capacitor voltage is relatively constant. Except capacitor dynamics, there is another pivotal dynamics in the conventional PLL. PLL dynamic can significantly affect the frequency response of the VS/CC-VSC, especially under the condition of weak grid [52-54]. Generally, the timescale of the PLL dynamic is approximately $1-30 \mathrm{~Hz}$ $[54,55]$, and it is close to the electromechanical timescale. Without the control loop reconstructions mentioned above, a virtual electromechanical swing process like SG in the VS/CC-VSC with a conventional synchronous reference frame PLL is still revealed [56].

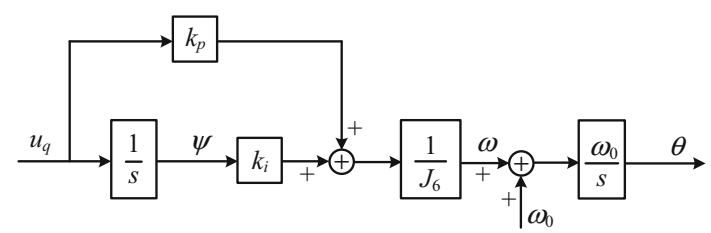

Fig. 18 Diagram of conventional PLL 
As shown in Fig. 18, the conventional synchronous reference frame PLL is given. $k_{p}$ and $k_{i}$ are the proportional and integral parameters of the PLL controller. The equivalent inertia parameter $J_{6}$ can be regarded as an common factor of the proportional and integral parameters. The status variable $\Psi$ in the PLL can be regarded as an indication of the energy level of the VS/CC-VSC, which is defined as the virtual energy. An SG-equivalent smallsignal model as shown in Fig. 1 is also derived in [56], utilizing the electromechanical dynamic of the conventional PLL. The corresponding inertia coefficient, synchronizing coefficient, and damping coefficient can be derived as the following:

$$
\left\{\begin{array}{l}
K_{J}=J_{6} \\
K_{S}=k_{i} U_{g} \cos \theta_{0} \\
K_{D}=k_{p} U_{g} \cos \theta_{0}
\end{array}\right.
$$

\subsection{SG-equivalent model of PS/CC-VSC}

As shown in Fig. 19, block diagram of the PS/CC-VSC is given. There is DG with fluctuant power on the DC side. Thus, it is necessary for the controller of the PS/CC-VSC to keep the DC-link voltage stable. The PS/CC-VSC is widely used in the ordinary photovoltaic applications and wind power applications without ES on the DC side.

To enhance the power system stability with the increasing penetration level of VSC-based doubly fed induction wind generator (DFIWG), a supplementary control loop is integrated to the PS/CC-VSC to reintroduce inertia response [57], and the kinetic energy stored in the rotating mass of the wind turbine blades is extracted to support the power system frequency. Besides inertial control, primary frequency control of VSC-based DFIWG is also proposed [58-60]. It can thereby participate in primary frequency control as traditional SG during an eventual decrease of system frequency under the premise of enough wind power. In [61], the authors presented an integrated control method achieving both the inertia property and primary frequency control. As shown in Fig. 20, the upper

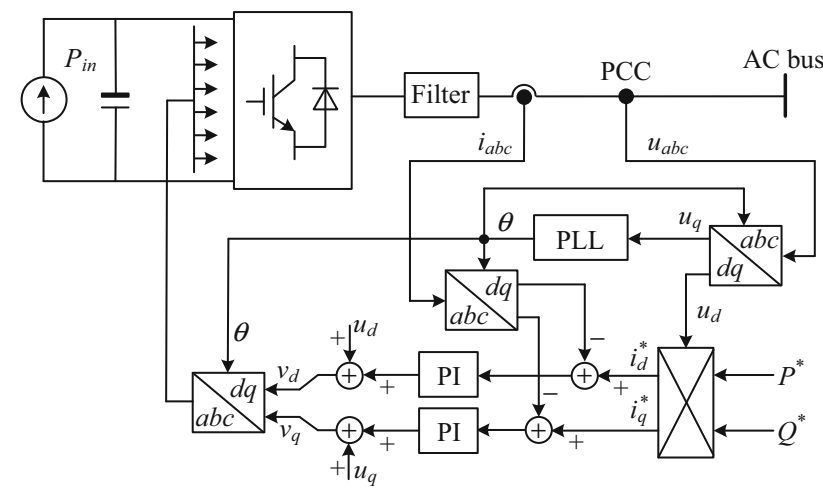

Fig. 19 Block diagram of PS/CC-VSC

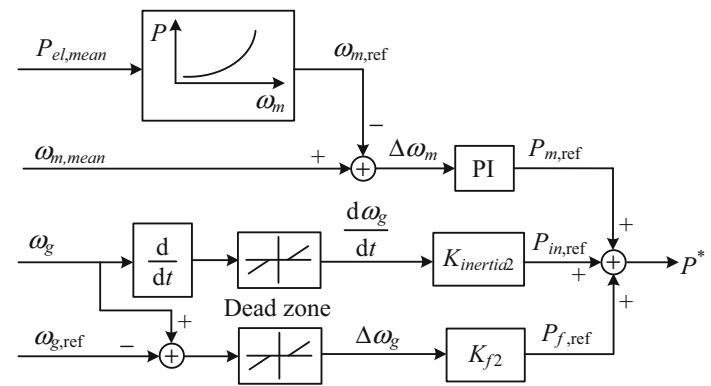

Fig. 20 Inertia emulating and primary frequency supporting control

branch is the basic rotor control loop, which is responsible for the general power balance of the DC-link. The middle branch is the inertia emulating control unit, and the last one is used for the primary frequency support.

Based on Fig. 20, the PS/CC-VSC can be integrated into the proposed modeling framework, and the corresponding inertia coefficient, synchronizing coefficient, and damping coefficient are given in (18), where $K_{f 2}$ and $K_{\text {inertia } 2}$ are the proportional gain coefficient and the differential gain coefficient respectively.

$$
\left\{\begin{array}{l}
K_{J}=K_{\text {inertia } 2} \\
K_{S}=\frac{3}{2} \frac{U_{1} U_{g}}{X_{L}} \cos \theta_{0} \\
K_{D}=K_{f 2}
\end{array}\right.
$$

Actually, inherent similarities of the physical meaning and internal mechanism between the PS/CC-VSC and the traditional SG system still exist even without the supplementary control loops as previously mentioned. Recently, several physical concepts related to PS/CCVSCs are developed [24], demonstrating the inherent unity of PS/CC-VSC and traditional SG. As mentioned in [24], the stability on electromechanical timescale of the PS/CCVSC mainly depends on the capacitor power balance and its voltage stability, as shown in Fig. 21. It is similar to the rotor angle stability of traditional SG system. There is a similar pattern between the dynamic behaviors of SG rotor and the DC capacitor. Taking advantage of the similarity, the equivalent physical mechanism compared to the $\mathrm{SG}$ is revealed and the SSG model is established [24]. The model

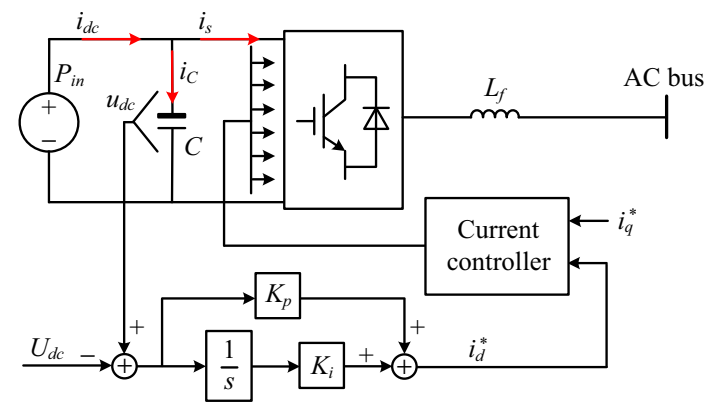

Fig. 21 DC capacitor voltage-control scheme of PS/CC-VSC 
can be expressed by the block diagram in Fig. 1, and the corresponding characteristic coefficients can be derived as the following [24]:

$$
\left\{\begin{aligned}
K_{J} & =\frac{C U_{d c}^{2}}{P_{b a s e}} \\
K_{S} & =\frac{3}{2} K_{i} U_{g} \cos \theta_{0} \\
K_{D} & =\frac{3}{2} K_{p} U_{g} \cos \theta_{0}
\end{aligned}\right.
$$

where $C$ is the DC capacitance; $U_{d c}$ is the rated voltage of the capacitor; $K_{p}$ and $K_{i}$ are the proportional and integral parameters of the $U_{d c}$ controller.

\section{Comparison of different VSCs}

Various VSCs can be categorized into four types from the perspective of application. There are PS/CC-VSCs, VS/ CC-VSCs, VS/VC-VSCs and PS/VC-VSCs, according to the types of energy supply on the DC side and the control modes on the AC side. As mentioned above, SG-imitation for all the VSCs can be implemented by constructing supplementary control loop or utilizing the inherent electromechanical swing dynamics. Though the specific procedures of SG-imitation for these VSCs are different, the classical Phillips-Heffron model can be applied in the synchronization stability investigation of various VSCs. A modeling framework is established for all kinds of VSCs to clarify the corresponding inertia, synchronizing and damping coefficients. Different from the traditional SGs, the equivalent inertia, synchronizing and damping coefficients of VSCs mentioned above are flexible and varied, as

Table 1 Comparison of the equivalent inertia and the synchronizing and damping coefficients of VSCs

\begin{tabular}{llll}
\hline Case & $K_{J}$ & $K_{S}$ & $K_{D}$ \\
\hline 1 & $k_{1} \Delta P / k_{P}$ & $1.5 U_{1} U_{g} \cos \left(\theta_{0}\right) / X_{L}$ & $1 / k_{P}$ \\
2 & $J_{1}$ & $1.5 U_{1} U_{g} \cos \left(\theta_{0}\right) / X_{L}$ & $D_{p 1}+1 / k_{P}$ \\
3 & $J_{2}$ & $1.5 U_{1} U_{g} \cos \left(\theta_{0}\right) / X_{L}$ & $D_{p 2}$ \\
4 & $T_{f} / k_{P}$ & $1.5 U_{1} U_{g} \cos \left(\theta_{0}\right) / X_{L}$ & $1 / k_{P}$ \\
5 & $T_{b} / k_{P}$ & $1.5 U_{1} U_{g} \cos \left(\theta_{0}\right) / X_{L}$ & $K_{S} T_{a}$ \\
6 & $J_{3}$ & $1.5 U_{1} U_{g} \cos \left(\theta_{0}\right) / X_{L}$ & $K_{u 1} K_{u 2}$ \\
7 & $C U_{d c}^{2} / P_{\text {base }}$ & $1.5 U_{1} U_{g} \cos \left(\theta_{0}\right) / X_{L}$ & $K_{S} K_{d 1}$ \\
8 & $J_{4}$ & $U_{g} \cos \left(\theta_{0}\right)$ & $K_{F}$ \\
9 & $K_{\text {inertia } 1}$ & $1.5 U_{1} U_{g} \cos \left(\theta_{0}\right) / X_{L}$ & $K_{f 1}$ \\
10 & $J_{5}$ & $1.5 U_{1} U_{g} \cos \left(\theta_{0}\right) / X_{L}$ & $K_{d 2}$ \\
11 & $J_{6}$ & $k_{i} U_{g} \cos \left(\theta_{0}\right)$ & $k_{p} U_{g} \cos \left(\delta_{0}\right)$ \\
12 & $K_{\text {inertia } 2}$ & $1.5 U_{1} U_{g} \cos \left(\theta_{0}\right) / X_{L}$ & $K_{f 2}$ \\
13 & $C U_{d c}^{2} / P_{\text {base }}$ & $1.5 K_{i} U_{g} \cos \left(\theta_{0}\right)$ & $1.5 K_{p} U_{g} \cos \left(\theta_{0}\right)$ \\
\hline
\end{tabular}

shown in Table 1. The 13 cases of SG-imitation for various VSCs shown in Table 1 are as follow: Case 1, flexible inertia loop for droop control; Case 2, virtual inertia frequency control; Case 3, VSG control; Case 4, SG model of droop control by LPF; Case 5, SG-imitation by lead-lag unit; Case 6, control considering the $u_{d c}$ dynamic; Case 7, SG-imitation by the DC-link; Case 8, reproduce PLL feature of SG; Case 9, VSYNC project; Case 10, VISMA; Case 11, equivalent SG model by the PLL; Case 12, improved DFIWG control; Case 13, SSG model by the DClink dynamic.

For VSCs without constant voltage-source supply on the DC side, namely the PS/CC-VSCs and PS/VC-VSCs, the DC-link has an inherent electromechanical swing dynamic. It can be utilized for SG-imitation, such as Cases 7 and 13 in Table 1. On this occasion, the equivalent inertia coefficient directly depends on the DC capacitance and the rated voltage of the DC-link. It gives a physical insight of the equivalent inertia. In terms of the VSCs without utilizing the DC-link dynamic directly, such as Cases 6 and 12, the equivalent inertia seems to be a flexible configuration. However, the constraints of the short-term power supply on the DC side must be considered when selecting inertia parameter as there is no ES. For those VSCs with ES, namely the VS/CC-VSCs and VS/VC-VSCs, the DC-link voltage almost maintains a constant. Their equivalent inertia can be freely configured according to the system requirements. It is obvious that the equivalent synchronizing coefficient of VC-VSCs is completely consistent with that of the traditional SGs, seen from Cases 1 to 7 . Nevertheless, as a current-source relying on the synchronization to a stable grid voltage, the equivalent synchronizing coefficient of most CC-VSCs is related to the parameters of the PLL or the reconstructed PLL.

\section{Future trends}

Along with an increase in VSC-based device penetration, there is an increasing concern over the frequency response and stability of the VSC-based modern power system. The concepts of the virtual rotor angle [25] and power-electronics-enabled power system [62, 63] are proposed successively. A solution toward enhancing the stability is to provide additional electromechanical dynamic as the traditional SG virtually. As mentioned above, various control strategies and analysis focus on the equivalence between the VSCs and SGs. Considerable efforts are still needed to reliably integrate large amounts of DGs into the power system. Here are some important further research trends:

Firstly, more effective and practical algorithms as well as control methodologies are needed to simulate the 
behaviors of SG, taking into account the different practical applications. The energy buffer that acts as a virtual rotor is the key for VSCs in SG-imitation, such as the DC-link. The selection of the energy buffer should be made according to specific circumstances for different VSC-based DGs. The utilization of the energy buffer by different control strategies will achieve different SG-equivalent dynamics. The time scale of the response, the power capacity and the duration of the energy are important indicators for the energy buffer. It is necessary for further studies to coordinate the timing and the capacity of the energy discharge of the VSC-based DGs with the characteristics of SGs.

Secondly, more attention needs to be paid to the potential flexibility of VSC-based devices when simulating the SG properties. For physical SG, the rotational momentum and friction factor cannot be changed, while it is easy for the VSCs to optimize the equivalent inertia or damping coefficient needed, providing that the timing and the capacity of the energy buffer is adequate. For example, some self-tuning methods allow the SG-equivalent VSC to increase and reduce its virtual inertia and virtual damping considering the acceleration/deceleration of the virtual angular velocity in each phase of oscillation $[64,65]$ in order to achieve better performance of the frequency response than that in conventional SGs.

Thirdly, greater clarity about the dissimilarity between various VSCs and SG needs to be pursued, based on the existing knowledge about the similarity and equivalence mentioned in this paper. A common sense, which needs be considered, is that the similarity between VSCs and SG is special while the dissimilarity is general, although many efforts have been made to seeking the equivalence between them. Only the limitations of the equivalence have been clearly understood, the equivalence can be better utilized. For example, the current limitation in droop-controlled VSCs makes it more prone to lose synchronous stability than conventional SGs [25]. Even more importantly, the VSCs possess more a complex impedance characteristic in the frequency band above the fundamental frequency, which may also cause super-synchronous resonance. This is also a difference from the traditional SG.

Fourthly, it is necessary to make intensive efforts toward the application of well-developed models, theories, and methods in traditional power system to the investigation of dynamic behaviors and stability of modern power system with VSC-based DGs. Based on the full knowledge of similarities and differences between SGs and various VSCbased DGs, modeling and analysis of VSC-based systems can be implemented by taking advantage of traditional theories, in some limited and appropriate occasions. For example, some valuable conclusions about synchronization and stability for microgrid with multiple VSCs are extracted by applying the classic Kuramoto oscillator theory that is originally utilized in a multi-SG system [44].

Finally, it is also necessary to develop new standards and certifications regarding the virtual inertia and damping for the VSC-based DGs, considering the frequency response requirements of power grid and specific situations of different VSC-based DGs synthetically. The previously mentioned discrimination of similarities and differences, and the analysis results from the perspective of traditional theory, can provide reference for the standards and certifications. This work is significant for coordinating operation between VSCs and SGs in the new environment with growing penetration of VSC-based devices.

\section{Conclusion}

This paper categorizes various VSCs into four types according to the energy supply on the DC side and the control modes on the AC side. There are PS/CC-VSCs, VS/ CC-VSCs, VS/VC-VSCs and PS/VC-VSCs. It is revealed that the SG-equivalent electromechanical swing dynamic can be extracted from all the VSCs. The key point on SGimitation is taking advantage of the real or virtual energy buffer in VSCs. Then, a complete framework of SGequivalent small-signal modeling of all kinds of VSCs is established. The VSCs with different application environments have similar dynamic behavior, and the behavior characteristics can be represented by the classical PhillipsHeffron model. Thus, the equivalent inertia, synchronizing coefficient, and damping coefficient in different VSCs are derived and compared. The modeling framework based on a comparison with a traditional SG is beneficial for clearly cognizing the VSC-based DGs. Finally, the future research trend is addressed. Some important topics are mentioned: improvement of effective and practical SG-equivalent strategies considering the particularity of different VSCbased DGs, performance improvement by exploiting the potential flexibility of VSC-based DGs, more thorough understanding of the dissimilarity, migrating application of modeling and analysis theories, and formulating or revising future standards for the new system.

Acknowledgments This work was supported by National High Technology Research and Development Program of China (No. 2015AA050606), National Key Research and Development Program (No. 2016YFB0900302) and National Natural Science Foundation of China (Nos. U1510208, 61273045, 51361135705).

Open Access This article is distributed under the terms of the Creative Commons Attribution 4.0 International License (http:// creativecommons.org/licenses/by/4.0/), which permits unrestricted use, distribution, and reproduction in any medium, provided you give appropriate credit to the original author(s) and the source, provide a 
link to the Creative Commons license, and indicate if changes were made.

\section{References}

[1] Liserre M, Sauter T, Hung JY (2010) Future energy systems: integrating renewable energy sources into the smart power grid through industrial electronics. IEEE Ind Electron Mag 4(1):18-37

[2] Blaabjerg F, Chen Z, Kjaer SB (2004) Power electronics as efficient interface in dispersed power generation systems. IEEE Trans Power Electron 19(5):1184-1194

[3] Flourentzou N, Agelidis VG, Demetriades GD (2009) VSCbased HVDC power transmission systems: an overview. IEEE Trans Power Electron 24(3):592-602

[4] Xu L, Fan L (2013) Impedance-based resonance analysis in a VSC-HVDC system. IEEE Trans Power Deliv 28(4):2209-2216

[5] Zhang L, Harnefors L, Nee HP (2010) Power-synchronization control of grid-connected voltage-source converters. IEEE Trans Power Syst 25(2):809-820

[6] Edris AA (1997) Proposed terms and definitions for flexible AC transmission system (FACTS). IEEE Trans Power Deliv 12(4):1848-1853

[7] Xue Y, Chang L, Kjaer SB et al (2004) Topologies of singlephase inverters for small distributed power generators: an overview. IEEE Trans Power Electron 19(5):1305-1314

[8] Liserre M, Teodorescu R, Blaabjerg F (2006) Stability of photovoltaic and wind turbine grid-connected inverters for a large set of grid impedance values. IEEE Trans Power Electron 21(1):263-272

[9] Lasseter RH (2002) Microgrids. In: Proceedings of the power engineering society winter meeting, New York, USA, 27-31 January 2002, 6 pp

[10] Chandorkar MC, Divan DM, Adapa R (1993) Control of parallel connected inverters in standalone AC supply systems. IEEE Trans Ind App 29(1):136-143

[11] Moon MS, Johnson RW (1999) DSP control of UPS inverter with over-current limit using droop method. In: Proceedings of the 30th annual IEEE power electron specialists conference, South Carolina, USA, 7-10 June 1999, 6 pp

[12] Chiang SJ, Chang JM (2001) Parallel control of the UPS inverters with frequency-dependent droop scheme. In: Proceedings of the power electronics specialists conference, Vancouver, Canada, 17-21 June 2001, 5 pp

[13] Ruttledge L, Miller NW, O’Sullivan J et al (2012) Frequency response of power systems with variable speed wind turbines. IEEE Trans Sustain Energy 3(4):683-691

[14] Eftekharnejad S, Vittal V, Heydt GT et al (2013) Impact of increased penetration of photovoltaic generation on power systems. IEEE Trans Power Syst 28(2):893-901

[15] Erdinc O, Paterakis NG, Catalão JPS (2015) Overview of insular power systems under increasing penetration of renewable energy sources: opportunities and challenges. Renew Sustain Energy Rev 52:333-346

[16] Yang Y, Enjeti P, Blaabjerg F et al (2013) Suggested grid code modifications to ensure wide-scale adoption of photovoltaic energy in distributed power generation systems. In: Proceedings of the industry applications society annual meeting, Florida, USA, 6-11 October 2013, 8 pp

[17] Majumder R (2013) Some aspects of stability in microgrids. IEEE Trans Power Syst 28(3):3243-3252

[18] Kundur P (1994) Power system stability and control. McGraw Hill, New York
[19] Jeon JH, Kim JY, Kim HM et al (2010) Development of hardware in-the-loop simulation system for testing operation and control functions of microgrid. IEEE Trans Power Electron 25(12):2919-2929

[20] Lee DJ, Wang L (2008) Small-signal stability analysis of an autonomous hybrid renewable energy power generation/energy storage system part I: time-domain simulations. IEEE Trans Energy Conversion 23(1):311-320

[21] Coelho EAA, Cortizo PC, Garcia PFD (2002) Small-signal stability for parallel-connected inverters in stand-alone AC supply systems. IEEE Trans Ind Appl 38(2):533-542

[22] Pogaku N, Prodanović M, Green TC (2007) Modeling, analysis and testing of autonomous operation of an inverter-based microgrid. IEEE Trans Power Electron 22(2):613-625

[23] Bottrell N, Prodanovic M, Green TC (2013) Dynamic stability of a microgrid with an active load. IEEE Trans Power Electron 28(11):5107-5119

[24] Xiong L, Zhuo F, Wang F et al (2016) Static synchronous generator model: a new perspective to investigate dynamic characteristics and stability issues of grid-tied PWM inverter. IEEE Trans Power Electron 31(9):6264-6280

[25] Xin H, Huang L, Zhang L et al (2016) Synchronous instability mechanism of $P-f$ droop-controlled voltage source converter caused by current saturation. IEEE Trans Power Syst 31(6):5206-5207

[26] Huang L, Zhang L, Xin H et al (2016) Current limiting leads to virtual power angle synchronous instability of droop-controlled converters. In: Proceedings of the power and energy society general meeting, Boston, USA, 17-21 July 2016, 5 pp

[27] Dorfler F, Bullo F (2012) Synchronization and transient stability in power networks and nonuniform Kuramoto oscillators. SIAM J Control Optim 50(3):1616-1642

[28] Heffron WG, Phillips RA (1952) Effect of a modern amplidyne voltage regulator on under excited operation of large turbine generators. Trans Am Inst Electr Eng 71(1):692-697

[29] Kundur P, Paserba J, Ajjarapu V et al (2015) Definition and classification of power system stability IEEE/CIGRE joint task force on stability terms and definitions. Golden Res Thoughts 4(8):1387-1401

[30] Loix T, Breucker SD, Vanassche P et al (2009) Layout and performance of the power electronic converter platform for the VSYNC project. In: Proceedings of the PowerTech, Bucharest, Romania, 28 June-2 July 2009, 8 pp

[31] Zhong Q, Weiss G (2011) Synchronverters: inverters that mimic synchronous generators. IEEE Trans Ind Electron 58(4):1259-1267

[32] Rocabert J, Luna A, Blaabjerg F et al (2012) Control of power converters in AC microgrids. IEEE Trans Power Electron 27(11):4734-4749

[33] Harada K, Katsuki A, Murata K et al (1985) On the parallel operations of triport UPS systems. In: Proceedings of the IEEE telecommunications energy conference, Amsterdam, Netherlands, 9-13 October 1985, 6 pp

[34] Chen JF, Chu CL, Huang CL (1992) The parallel operation of two UPS by the coupled-inductor method. In: Proceedings of the IEEE international symposium on industrial electronics, $\mathrm{Xi}$ ' an, China, 25-29 May 1992, 4 pp

[35] Vassilakis A, Kotsampopoulos P, Hatziargyriou N et al (2013) A battery energy storage based virtual synchronous generator. In: Proceedings of the 2013 IEEE IREP symposium, Rethymnon, Greece, 25-30 August 2013, 6 pp

[36] Ashabani M, Mohamed ARI (2014) Novel comprehensive control framework for incorporating VSCs to smart power grids using bidirectional synchronous-VSC. IEEE Trans Power Syst 29(2):943-957 
[37] Soni N, Doolla S, Chandorkar MC (2013) Improvement of transient response in microgrids using virtual inertia. IEEE Trans Power Deliv 28(3):1830-1838

[38] Gao F, Iravani MRA (2008) Control strategy for a distributed generation unit in grid-connected and autonomous modes of operation. IEEE Trans Power Deliv 23(2):850-859

[39] Sakimoto K, Miura Y, Ise T (2011) Stabilization of a power system with a distributed generator by a virtual synchronous generator function. In: Proceedings of the international conference on power electronics and ECCE Asia, Jeju, the Republic of Korea, 1-4 January 2011, 8 pp

[40] Chen Y, Hesse R, Turschner D et al (2011) Dynamic properties of the virtual synchronous machine (VISMA). In: Proceedings of the renewable energies and power quality conference, Valencia, Spain, 28-30 March 2011, 5 pp

[41] D'Arco S, Suul JA (2014) Equivalence of virtual synchronous machines and frequency-droops for converter-based microgrids. IEEE Trans Smart Grid 5(1):394-395

[42] Guan M, Pan W, Zhang J et al (2015) Synchronous generator emulation control strategy for voltage source converter (VSC) stations. IEEE Trans Power Syst 30(6):3093-3101

[43] Liu J, Miura Y, Ise T (2016) Comparison of dynamic characteristics between virtual synchronous generator and droop control in inverter-based distributed generators. IEEE Trans Power Electron 31(5):3600-3611

[44] Simpson-Porco JW, Dörfler F, Bullo F (2013) Synchronization and power sharing for droop-controlled inverters in islanded microgrids. Automatica 49(9):2603-2611

[45] Ashabani M (2014) Synchronous converter and synchronousVSC-state of art of universal control strategies for smart grid integration. In: Proceedings of the smart grid conference, Venice, Italy, 3-6 November 2014, 8 pp

[46] Ashabani M, Mohamed ARI (2014) New family of microgrid control and management strategies in smart distribution gridsanalysis, comparison and testing. IEEE Trans Power Syst 29(5):2257-2269

[47] Liu H, Chen Z (2015) Contribution of VSC-HVDC to frequency regulation of power systems with offshore wind generation. IEEE Trans Energy Conv 30(3):918-926

[48] Li Y, Yang Y, Liu G et al (2014) Coordinated control of wind farms and VSC-HVDC to improve inertia level of power system. Proc CSEE 34(34):6021-6031

[49] Cvetkovic I, Boroyevich D, Burgos R et al (2013) Synchronous generator-based grid-interface converter for energy storage systems integration. In: Proceedings of the grand challenges on modeling and simulation conference, Haikou, China, 20-24 December 2013, 6 pp

[50] Driesen J, Visscher K (2008) Virtual synchronous generators. In: Proceedings of the power and energy society general meeting, Pittsburgh, USA, 20-24 July 2008, 3 pp

[51] Beck HP, Hesse R (2007) Virtual synchronous machine. In: Proceedings of the elect power quality and utilisation conference, Barcelona, Spain, 10-13 October 2007, 6 pp

[52] Xi X, Geng H, Yang G (2014) Enhanced model of the doubly fed induction generator-based wind farm for small-signal stability studies of weak power system. IET Renew Power Gener 8(7):765-774

[53] Li R, Geng H, Yang G (2016) Fault ride-through of renewable energy conversion systems during voltage recovery. J Mod Power Syst Clean Energy 4(1):28-39

[54] $\mathrm{Hu}$ J, Hu Q, Wang B et al (2016) Small signal instability of PLL-synchronized type-4 wind turbines connected to highimpedance AC grid during LVRT. IEEE Trans Energy Convers 31(4):1676-1687

[55] Wen B, Boroyevich D, Burgos R et al (2015) Small-signal stability analysis of three-phase AC systems in the presence of constant power loads based on measured $d-q$ frame impedances. IEEE Trans Power Electr 30(10):5952-5963

[56] Tan S, Geng H, Yang G (2018) Phillips-Heffron model for current-controlled power electronic generation unit. J Mod Power Syst Clean Energy 6(3):582-594

[57] Ekanayake J, Jenkins N (2005) Comparison of the responce of doubly fed and fixed speed induction generator wind turbines to changes in network frequency. IEEE Trans Energy Conv 19(4):1277-1287

[58] Almeida RGD, Lopes JAP (2005) Primary frequency control participation provided by doubly fed induction wind generators. In: Proceedings of the 15th power system computation conference, Liege, Belgium, 22-26 August 2005, 7 pp

[59] Almeida RGD, Lopes JAP (2007) Participation of doubly fed induction wind generators in system frequency regulation. IEEE Trans Power Syst 22(3):944-950

[60] Morren J, Haan SWHD, Ferreira JA (2006) Contribution of DG units to primary frequency control. Eur Trans Elect Power 16(5):507-521

[61] Morren J, Haan SWHD, Kling WL et al (2006) Wind turbines emulating inertia and supporting primary frequency control. IEEE Trans Power Syst 21(1):433-434

[62] Salmani M, Rahbari-Asr N, Edrington CS et al (2016) Online and offline stability analysis methods for the power electronicbased components in design and operational stages. IEEE Trans Power Electron 31(4):3151-3164

[63] Kwasinski A (2011) Advanced power electronics enabled distribution architectures: design, operation, and control. In: Proceedings of the power electronics and ECCE Asia, Jeju, the Republic of Korea, 30 May-3 June 2011, 8 pp

[64] Alipoor J, Miura Y, Ise T (2015) Power system stabilization using virtual synchronous generator with alternating moment of inertia. IEEE J Emerg Sel Top Power Electron 3(2):451-458

[65] Miguel ATL, Lopes LAC, Luis AMT et al (2014) Self-tuning virtual synchronous machine: a control strategy for energy storage systems to support dynamic frequency control. IEEE Trans Energy Conv 29(4):833-840

Shulong TAN received the B.E. degree in automation from the Huazhong University of Science and Technology, Wuhan, China, in 2012. He is presently working towards the Ph.D. degree at Tsinghua University, Beijing, China. He is also working as a research assistant in the Department of Automation, Tsinghua University. His current research interests include the stability problems of inverter-based renewable power generation systems.

Hua GENG received the B.S. degree in electrical engineering from the Huazhong University of Science and Technology, Wuhan, China, in 2003, and the Ph.D. degree in control theory and application from Tsinghua University, Beijing, China, in 2008. From 2008 to 2010, he was a postdoctoral research fellow in the Department of Electrical and Computer Engineering, Ryerson University, Toronto, Canada. Since May 2010, he has been an assistant professor in the Department of Automation, Tsinghua University. His current research interests include distribution generation systems, renewable energy conversion systems, and digital control systems.

Geng YANG received the B.S. and M.S. degrees in electrical engineering from the Xi'an University of Science and Technology, Xi'an, China, in 1982 and 1984, respectively, and the Ph.D. degree in electrical engineering from Sophia University, Chiyoda, Japan, in 1992. From 1985 to 1987 , he was an assistant at the Xi'an University of Science and Technology. He was a visiting scientist with Fukui State University, Fukui, Japan, in 1987, and with Sophia University, 
Tokyo, Japan, in 1988. From 1992 to 1994, he was a senior researcher with Kasuga Electrical Works Ltd., Mitaka, Japan. From 1995 to 1999, he was a lecturer, associate professor, and professor with the Xi' an University of Science and Technology. Since 2000, he has been a professor in the Department of Automation, Tsinghua University, Beijing, China. His current research interests include electrical drives and systems, power electronic equipment, and the control technology of wind and photovoltaic energy conversion systems.

Huai WANG received the B.E. degree in electrical and electronic engineering from the Huazhong University of Science and Technology, Wuhan, China, in 2007, and the Ph.D. degree in electronic engineering from the City University of Hong Kong, Kowloon, Hong Kong, in 2012. He has been with Aalborg University, Aalborg, Denmark, since 2012, where he is currently an assistant professor with the Department of Energy Technology. His current research interests include the reliability of DC-link capacitors, reliability of power electronic systems, high-voltage DC-DC power converters, time-domain control of converters, and passive components reduction technologies.

Frede BLAABJERG received the Ph.D. degree from Aalborg University, Aalborg, Denmark, in 1992. From 1987 to 1988, he was with ABB-Scandia, Randers, Denmark. He is with Aalborg University, where he became an assistant professor in 1992, an associate professor in 1996, and a full professor of power electronics and drives in 1998. He has been a part time research leader with the Research Center Risoe in wind turbines. From 2006 to 2010, he was the dean of the Faculty of Engineering, Science, and Medicine and became a visiting professor with Zhejiang University, Hangzhou, China, in 2009. He received the IEEE PELS Distinguished Service Award in 2009, the EPE-PEMC Council Award in 2010 and the IEEE William E. Newell Power Electronics Award in 2014. He was the editor-inchief of the IEEE Transactions on Power Electronics from 2006 to 2012. His current research interests include power electronics and its applications such as in wind turbines, PV systems, reliability, harmonics, and adjustable-speed drives. 